\title{
Estimation of Uncertainty in the Flow Field around Underground Ains (Qanats) Using Monte-Carlo Method
}

\author{
Amro M. M. Elfeki \\ Water Resources Dept., Faculty of Meteorology, Environment and Arid \\ Land Agriculture, King Abdulaziz University, \\ Jeddah, Saudi Arabia \\ amro_elfeki@yahoo.co.uk
}

\begin{abstract}
Subsurface is heterogeneous in its structure. Its heterogeneity makes predictions very complicated. Recently, researchers have developed techniques to incorporate heterogeneity on models to be able to quantify the heterogeneity and to study its effect on engineering structures. Numerical experiments were designed, through the development of a computer code called "FLOW2AIN", to simulate groundwater flow to Ains (Qanats) in a heterogeneous subsurface. The purpose of these experiments is to quantify the effect of uncertainty in the local heterogeneity around the Ain on the flow field and the expected flux of water infiltrated to the Ain from the ambient groundwater. The numerical experiments discuss the influence of the degree of heterogeneity on the expected seepage discharge towards the Ain and its uncertainty due to heterogeneity of the subsurface.
\end{abstract}

\section{Introduction}

Estimation of the amount of water provided by Ains is important for the sustainable development of irrigation projects and drinking water issues. Reliable estimation of this amount is subjected to many factors such as the groundwater conditions at the site of the Ain, the surface land use, the heterogeneity of the subsoil at the location of the Ain, etc. One of the factors that we will focus on in this study is the heterogeneity of the subsoil. 
Subsurface is heterogeneous in its structure. Many researchers have observed these heterogeneity (Gelhar, 1986; Sudicky, 1986; Dagan, 1989; and Hess et al., 1992). This complex structure of geological formations has attracted considerable attention from geostatisticians, hydro-geologists, oil reservoir engineers, mining engineers, and others who are interested in geosciences to evaluate the effect of heterogeneity on groundwater studies and subsurface infrastructures. The literature on techniques describing heterogeneity is abundant and rapidly increasing (Gelhar. 1986, and Dagan 1989). In the next section, a review is made on some techniques.

In this paper, a model called FLOW2AIN has been developed to study the effect of heterogeneity, in terms of the hydraulic conductivity around Ain, which is modeled as a random space function (random field) described by its mean, variance and its correlation structure on the hydraulic head field around Ains and the quantity of water flux seeping into Ains (Fig. $1 \& 2$ ). The model is able to simulate the flow pattern around Ain under various degrees of heterogeneity of the subsurface and computes the seepage discharge towards Ain. The model cannot only be used to estimate the quantity of water provided by Ains but also it provides an estimate of uncertainty in the expected discharge through Ain.

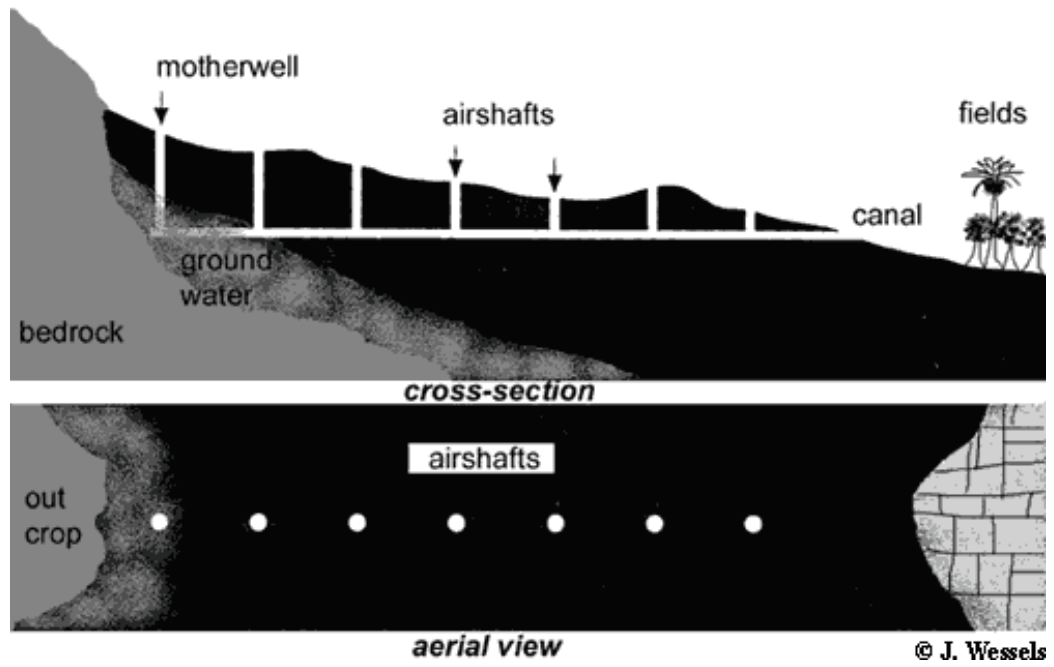

Fig. 1. Typical Ain longitudinal section and aerial view. 


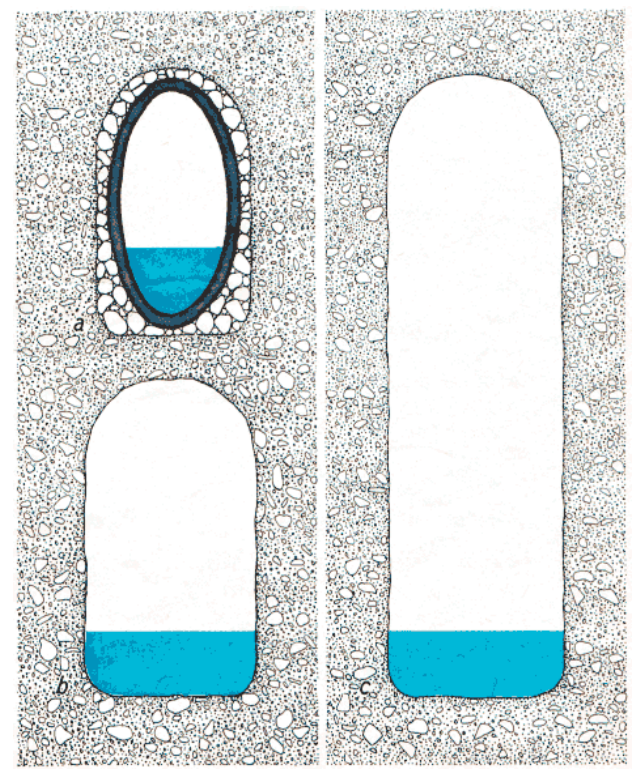

Fig. 2. Typical Ain cross-sections.

\section{Review of Literature and Theoretical Background}

Methods that generate random fields of hydrological parameters are immense (Rouhani \& Hall, 1988 and Park et al., 2005). The method of covariance matrix is one of the common methods in literature. For the mathematical theory books on statistical methods and analysis such as Ross, 2000, can be reviewed. The algorithm for generating random fields with a given covariance structure based on the covariance matrix of the system is explained in details by Elfeki, 1996. This method is going to be used in this paper. A more detailed explanation is given in the next section. Another method used for generating a correlated random field is called "Nearest Neighbor Method". This method is sometimes called Whittle Model. The idea of this method is based on generating an independent random field. Then, the value of a parameter at a given node is replaced by taking weighted average of the previous random values at the given node and a few surrounding ones. This model was first applied to one-dimensional steady state groundwater flow in a bounded domain by Smith \& Freeze (1979 a), and then they also extended the same approach to the two-dimensional steady state groundwater flow in Smith \& Freeze (1979 b). 
Elfeki \& Dekking (2001) have developed a technique to handle heterogeneity. The method they used is based on Markov chains. A brief description of the coupled Markov chain model is given below. The model is stochastic in nature, and couples two Markov chains. The first one is used to describe the sequence of lithologies in the vertical direction, and the second describes the sequence in the lithological structure in the horizontal direction.

Monte-Carlo approach is a widely used method to evaluate the effect of heterogeneity on groundwater flow problems. The idea of the method is based mainly on generating random fields of the hydrogeological parameters to represent the heterogeneity of the formation by the methods described above. Then, usual groundwater flow can be solved numerically in this geometrical structure. One can assume the probability density function of the model parameters or joint probability density function for a number of parameters in the model. The assumptions of these density functions are based on some field tests and/or laboratory tests. By using a random number generator, one generates a realization for each of these parameters. The parameter generation can be correlated or uncorrelated depending on the type of the problem. With this parameter, a classical numerical flow and/ or transport model is run and a set of results is obtained; another random selection of the parameters is made and the model is run again, and so on. It is necessary to have a very large number of runs, and the output model results corresponding to each input is obtained. Statistical analysis of the ensemble of the output can be done to get the mean, variance, covariance or the probability density function for each node with a location in the grid.

\section{Methodology}

The hydrodynamic condition of the flow system in this study is a steady state groundwater flow in a non-deformable saturated heterogeneous porous medium which according to Bear (1972), is governed by:

$$
\nabla \cdot[K(\mathbf{x}) \nabla \Phi(\mathbf{x})]=0,
$$

where $K(\mathbf{x})$ is the hydraulic conductivity, and $\Phi(\mathbf{x})$ is the hydraulic head at location $\mathbf{x}$. 
Figure 3 shows a schematic representation of the flow problem considered in this study. The numerical experiments are carried out using a model of a generic Ain and the ambient groundwater system. Dimensions of the model domain and the parameters that have been used are chosen to reflect conditions of a typical Ain. The model domain is defined by $0 \leq x \leq 50 \mathrm{~m}$ and $-20 \leq \mathrm{y} \leq 0 \mathrm{~m}$ (Fig. 4). The model is discretized with grid cells of $1 \mathrm{~m}$ by $1 \mathrm{~m}$ in both $\mathrm{x}$ - and $\mathrm{y}$-directions. The flow equation is solved numerically using a finite difference scheme (for details see Kinzelbach, 1986; Ababou et al., 1989; and Elfeki, 1996).

The stochastic simulation, followed in this paper, is based on MonteCarlo method (Issak, 1990; Smith \& Freeze, 1979 a \& b). A generation of hydraulic conductivity structure is first generated using the method of lower-upper (LU) decomposition (Elfeki, 1996) with data displayed in Table 1. The generated realization is called a realization of the subsurface. Then, on that realization, the groundwater flow problem is then solved under specified boundary conditions (Fig. 3). The solution leading to the hydraulic head corresponds to that realization. Another realization is generated and the flow model is run again, and so on up to the total number of realizations specified by the user. Statistical analysis of the ensemble of the output realizations can be carried out to obtain the expected value and variance of the output variables at each node in the grid.

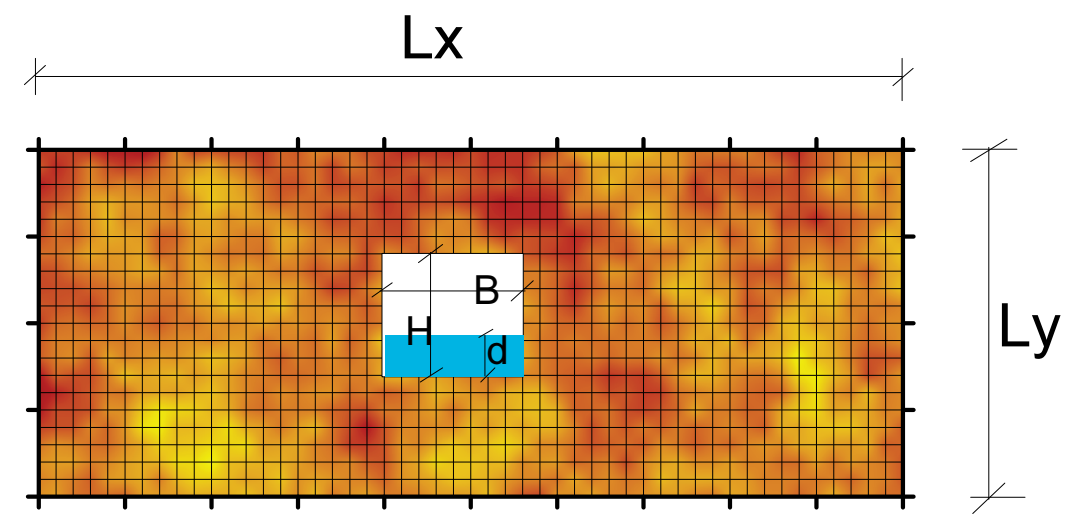

Fig. 3. Domain discretization and dimensioning of the problem.

Trial numerical experiments have been performed to study the accuracy of the flow model under the given hydraulic conductivity contrasts. The criterion for convergence in the solution of the problem is that the sum of residuals should be small enough. It has been shown that 
an accuracy limit of 0.0001 is reasonable in terms of stable results and computational costs. 1000 Monte-Carlo realizations are used to study the convergence of the computations. Table 1 shows the flow parameters used in the numerical experiments. In the Monte-Carlo runs, the mean head fields are constructed by averaging over the total number of realizations. Ensemble average hydraulic head $\langle\Phi(\mathbf{x})\rangle$ can be determined at each grid point as:

$$
\langle\Phi(\mathbf{x})\rangle=\frac{1}{M C} \sum_{k=1}^{M C} \Phi_{k}(\mathbf{x}),
$$

where, $\Phi_{k}(\mathbf{x})$ is the hydraulic head at location $\mathbf{x}$ in the $k^{\text {th }}$ realization of Monte-Carlo runs (MC).

In real field situation, however, there is always a single (actual) realization. Therefore, one could expect to find the actual head within the $95 \%$ significant level. The head variance at each grid point is given by:

$$
\sigma_{\Phi}^{2}(\mathbf{x})=\frac{1}{M C} \sum_{k=1}^{M C}\left[\Phi_{k}(\mathbf{x})-\langle\Phi(\mathbf{x})\rangle\right]^{2}
$$

$\sigma_{\Phi}^{2}(\mathbf{x})$ represents the uncertainty in the predictions.

Table 1. Numerical simulation values used in the numerical flow experiment of the program (FLOW2AIN).

\begin{tabular}{|l|l|}
\hline Parameter & Numerical Value \\
\hline Geometric mean of hydraulic conductivity & $1 \mathrm{~m} /$ day \\
\hline Variance of Ln $(\mathrm{K})$ & $0.1,0.5,1.0,1.5,2$ \\
\hline Correlation length in both directions & $2 \mathrm{~m}$ \\
\hline No of Monte-Carlo & 1000 \\
\hline Domain dimensions & $\mathrm{Lx}=50 . \mathrm{m}, \mathrm{Ly}=20 . \mathrm{m}$ \\
\hline Domain discretezation & $\Delta \mathrm{x}=1 \mathrm{~m}, \Delta \mathrm{y}=1 \mathrm{~m}$ \\
\hline Water table elev. in the ambient groundwater & $1.0 \mathrm{~m}$ \\
\hline Accuracy of computations & 0.00001 \\
\hline Water surface elevation in Ain & $0 . \mathrm{m}$ \\
\hline Ain dimensions & $\mathrm{H}=5 \mathrm{~m} . \mathrm{B}=5 \mathrm{~m}$ \\
\hline
\end{tabular}




\section{Discussion}

Figure 4 shows the simulation results of the numerical experiments in terms of flow field and uncertainty in the hydraulic head field. In the rightmost column, the hydraulic head field is superimposed over a hydraulic conductivity realization at variance of $\operatorname{Ln}(\mathrm{K})=0.5,1.5,2$ respectively (from top to bottom). The hydraulic head field shows increase of irregularity of the hydraulic head contours due to the increase of the heterogeneity of the medium. White spots are high permeable zones and dark areas are low permeable zones.

The middle column in Fig. 4 shows the ensemble hydraulic head field. It is obvious that the ensemble head field is almost constant over all realizations because the geometric mean conductivity is the same for all realization which is the equivalent hydraulic conductivity of the medium in that case (Dagan, 1989).

Finally, the last column in Fig. 4 shows the variance in the hydraulic head field (the measure of the uncertainty). It is obvious that the uncertainty in the hydraulic head field increases with an increase of the heterogeneity of the medium as the figures go from top to bottom. The reason is that increasing heterogeneity leads to increase in uncertainty of the hydraulic head field.

Figure 5 shows the simulation results of the numerical experiments in terms of expected flux towards Ain and the uncertainty in the hydraulic head profiles.

Figure 5 (top left corner) shows the expected auto-correlation function over the realizations that show exponential decay correlation with lag distance. It confirms the autocorrelation model incorporated in the program.

The top right corner of Fig. 5 shows the hydraulic head variance at various degrees of variability. It is obvious that increasing heterogeneity is reflected in the hydraulic head variance. The maximum head variance increases from about 0.0005 up to 0.035 when heterogeneity varies from 0.1 up to 2. It is also clear from the figure that the uncertainty in the head profile has its maximum between the given head boundary at the Ain interface and the groundwater divide at the left and right boundaries. The uncertainty decreases going towards Ain, the head is known deterministically and towards the groundwater divide where the head variability is very small. 

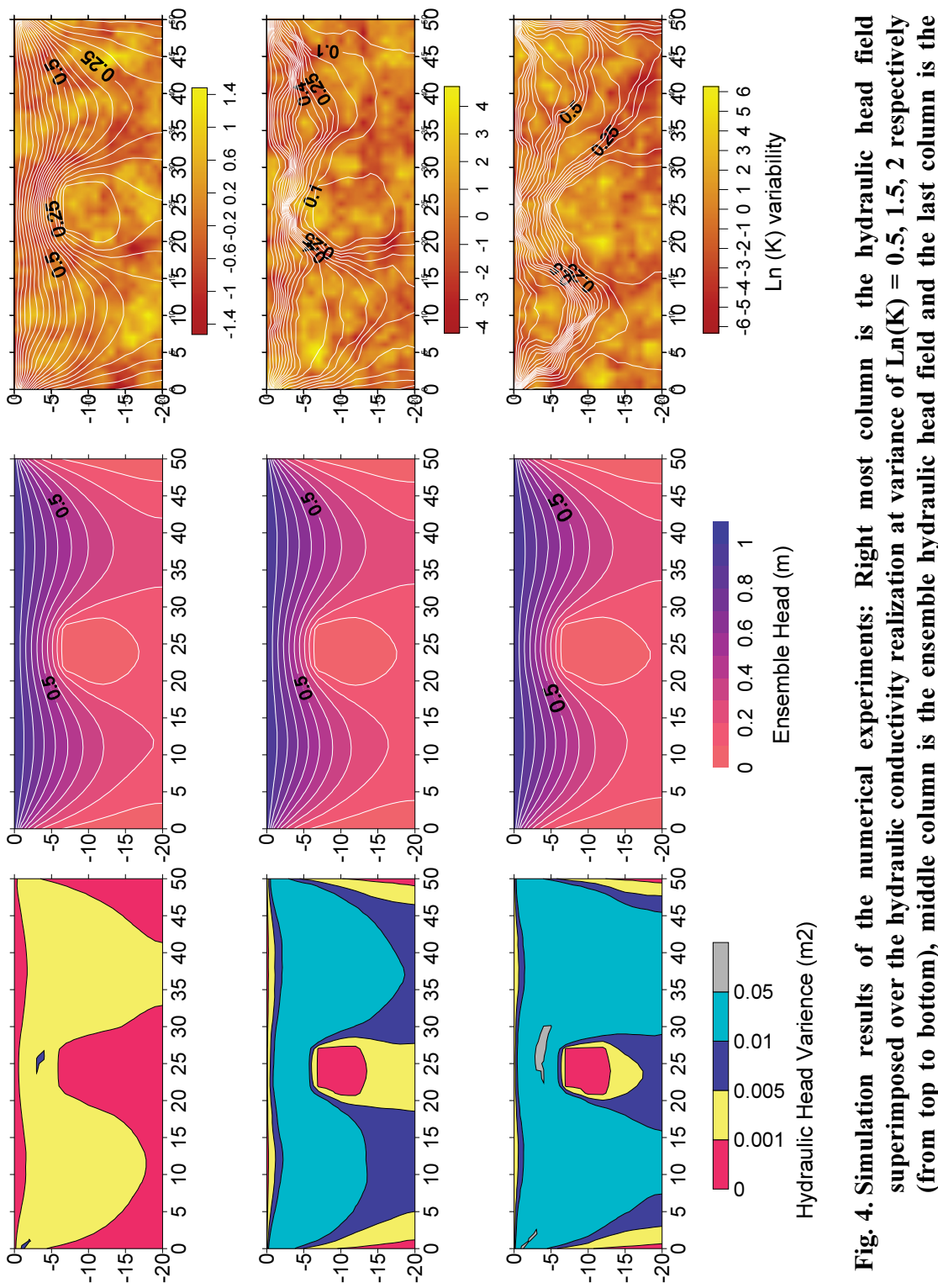

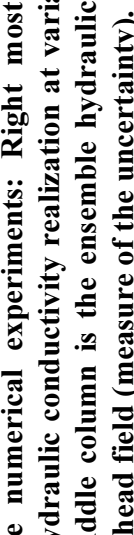
三. 

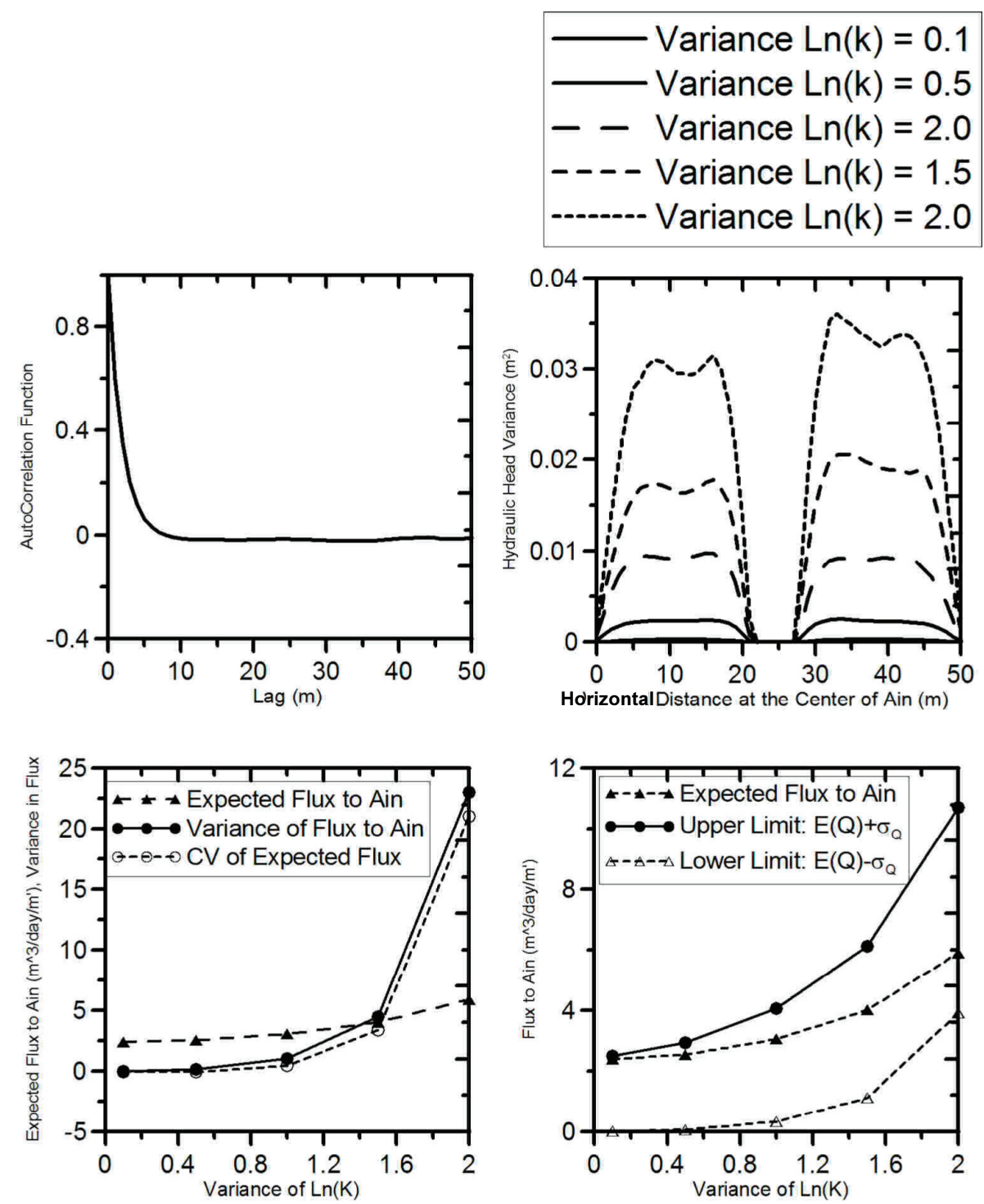

Fig. 5. Simulation results of the numerical experiments: Top left corner is the autocorrelation function. Top right corner is the hydraulic head variance at various degrees of variability. Left bottom corner is the Expected Flux to Ain, its variance and CV. Right bottom corner is the expected flux bounds.

The left bottom corner of Fig. 5 shows the expected flux to the Ain, its variance and coefficient of variation, CV. Increasing the degree of heterogeneity leads to an increase in the expected water flux towards the Ain, variance in flux and coefficient of variation due to the increase of 
variability of the hydraulic conductivity that samples more permeable zones as a result of the asymmetry of the lognormal density function.

The right bottom corner of Fig. 5 shows the expected flux bounds of the flow towards Ain. It provides the significant levels of the expected fluxes at various degree of heterogeneity. One would expect to have the flux to Ain within these bounds.

\section{Conclusions}

FLOW2AIN computer code has been developed to study the influence of flow variability around underground Ains on the quantity of water discharging to Ains. The results show the influence of soil variability on the expected head and the expected water flux entering the Ain at various heterogeneity conditions. Increasing heterogeneity of the hydraulic conductivity leads to an increase in the hydraulic head uncertainty and an increase in the water discharge into Ain. The increase in the expected water flux is due to the increase of the hydraulic conductivity variability as a result of increasing heterogeneity around Ain. The maximum head variance increases from about 0.0005 to 0.035 when heterogeneity varies from 0.1 to 2 . The model is going to be extended to handle more practical situations for predictions at field conditions. Ain Zubidah case study, at Makkah Al-Mokaramah region, Kingdom of Saudi Arabia, is foreseen.

\section{References}

Ababou, R., McLaughlin, D., Gelhar, L.W. and Tompson, A.F.B. (1989) Numerical simulation of three-dimensional saturated flow in randomly heterogeneous porous media, Transport in Porous Media, 4(6): 549-565.

Bear, J. (1972) Dynamics of Fluid in Porous Media, New York: American Elsevier.

Dagan, G. (1989) Flow and transport in Porous Formations, Springer, Verlag, New York.

Elfeki, A.M.M. (1996) Stochastic Characterization of Geological Heterogeneity and Its Impact on Groundwater Contaminant Transport, Ph.D. Diss, Delft University of Technology, Delft, The Netherlands.

Elfeki, A.M.M. and Dekking, M. (2001) A Markov chain model for subsurface characterization: Theory and applications, Mathematical Geology., 33(5): 569-589.

Gelhar, L.W. (1986) Stochastic subsurface hydrology from theory to applications, Water Resources Research, 22(9): 135-145.

Hess, K.M., Wolf, S.H. and Celia, M.A. (1992) Large-scale natural gradient tracer test in sand and gravel, Cape Cod, Massachusetts, 3 Hydraulic conductivity and calculated macrodispersivities, Water Resour. Res., 28: 2011-2027. 
Isaak, E. (1990) The Application of Monte Carlo Methods to the Analysis of Spatially Correlated Data, Ph.D. Dissertation, Stanford University, Stanford, California.

Kinzelbach, W. (1986) Groundwater modeling, Development in Water Science, 25: 333.

Park, E., Elfeki, A.M.M. and Dekking, M. (2005) Characterization of Subsurface Heterogeneity: Integration of Soft and Hard Information using Multidimensional Coupled Markov Chain Approach, Underground Injection Science and Technology Conference, Berkeley, 52.

Smith, L. and Freeze, A. (1979a) Stochastic analysis of steady state groundwater flow in a bounded domain, 1. One-dimensional simulations, Water Resour. Res., 15(3): 521-528.

Smith, L. and Freeze, A. (1979b) Stochastic analysis of steady state groundwater flow in a bounded domain, 2. Two-dimensional simulations, Water Resour. Res., 15(6): 1543-1559.

Sudicky, E.A. (1986) A natural gradient experiment on solute transport in a sand aquifer: Spatial variability of hydraulic conductivity and its role in the dispersion process, Water Resour. Res., 22(13): 2069-2083.

Ross, S. (2000) Introduction to Probability Models, 7th ed., Academic Press, San Diego, California, $693 \mathrm{p}$.

Rouhani, S. and Hall, T.J. (1988) Geostatistical schemes for groundwater sampling, Journal of Hydrology, 81(1): 85-102. 


\section{تقدير الريبة في حقل السريان حول العيون (القنوات) الجوفية باستخدام طريقة مونت كارلو}

\section{عمرو محمد الفقي \\ قسم علوم وإدارة موارد المباه، كلية الأرصاد والبيئة وزراعة الدناطق الجافة، جامعة الملك عبدالعزبز، جـدة - المدلكة العربية السعودية}

المستخلص. يعتبر سطح الأرض غيــر متجــانس فــي تكويناتــه

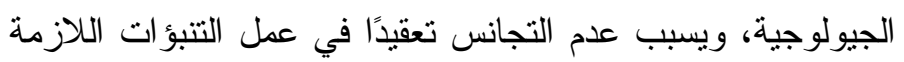

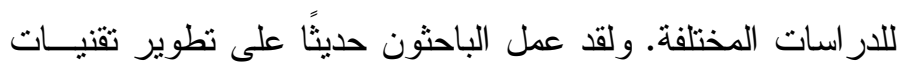

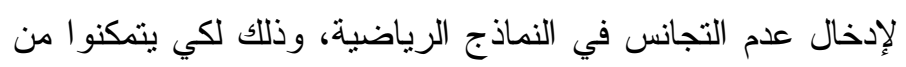
دراسة تأثنير عدم التجانس على المنشآت الهندسية.

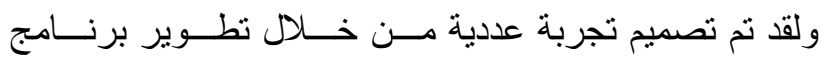

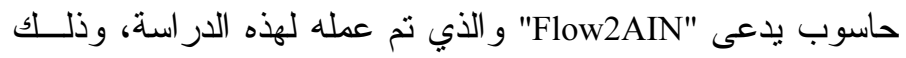

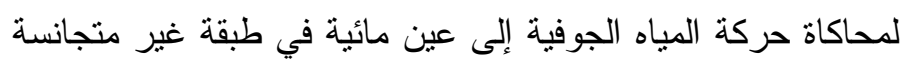

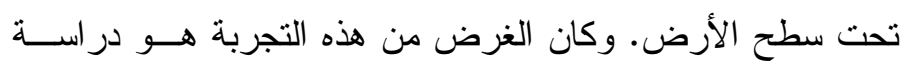

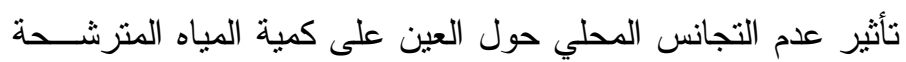

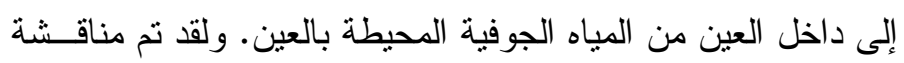

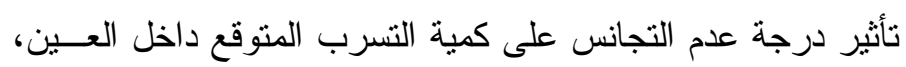

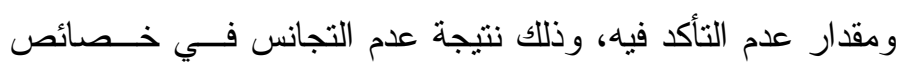

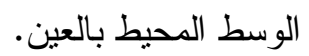

